\title{
Separation surgery for metastatic epidural spinal cord compression: comparison of a minimally invasive versus open approach
}

\author{
*Murray Echt, MD, 1,2 Ariel Stock, MD, PhD, 1,2 Rafael De la Garza Ramos, MD,,2 \\ Evan Der, PhD, ${ }^{1}$ Mousa Hamad, MD,1,2 Ryan Holland, MD,,2 Phillip Cezayirli, MD,,,2 \\ Rani Nasser, MD, ${ }^{3}$ Vijay Yanamadala, MD, MBA, ${ }^{1,2}$ and Reza Yassari, MD, MS ${ }^{1,2}$ \\ ${ }^{1}$ Spine Research Group and ${ }^{2}$ Department of Neurological Surgery, Montefiore Medical Center/Albert Einstein College of Medicine, \\ Bronx, New York; and 'Department of Neurological Surgery, University of Cincinnati College of Medicine, Cincinnati, Ohio
}

OBJECTIVE The aim of this study was to compare outcomes of separation surgery for metastatic epidural spinal cord compression (MESCC) in patients undergoing minimally invasive surgery (MIS) versus open surgery.

METHODS A retrospective study of patients undergoing MIS or standard open separation surgery for MESCC between 2009 and 2019 was performed. Both groups received circumferential decompression via laminectomy and a transpedicular approach for partial corpectomy to debulk ventral epidural disease, as well as instrumented stabilization. Outcomes were compared between the two groups.

RESULTS There were 17 patients in the MIS group and 24 in the open surgery group. The average age of the MIS group was significantly older than the open surgery group ( 65.5 vs 56.6 years, $p<0.05$ ). The preoperative Karnofsky Performance Scale score of the open group was significantly lower than that of the MIS group, with averages of $63.0 \%$ versus $75.9 \%$, respectively $(p=0.02)$. This was also evidenced by the higher proportion of emergency procedures performed in the open group ( 9 of 24 patients vs 0 of 17 patients, $p=0.004$ ). The average Spine Instability Neoplastic Score, number of levels fused, and operative parameters, including length of stay, were similar. The average estimated blood loss difference for the open surgery versus the MIS group ( $783 \mathrm{~mL}$ vs $430 \mathrm{~mL}, p<0.05$ ) was significant, although the average amount of packed red blood cells transfused was not significantly different ( $325 \mathrm{~mL} v 216 \mathrm{~mL}, p=0.39$ ). Time until start of radiation therapy was slightly less in the MIS than the open surgery group (32.8 \pm 15.6 days vs $43.1 \pm$ 20.3 days, $p=0.069$ ). Among patients who underwent open surgery with long-term follow-up, $20 \%$ were found to have local recurrence compared with $12.5 \%$ of patients treated with the MIS technique. No patients in either group developed hardware failure requiring revision surgery.

CONCLUSIONS MIS for MESCC is a safe and effective approach for decompression and stabilization compared with standard open separation surgery, and it significantly reduced blood loss during surgery. Although there was a trend toward a faster time to starting radiation treatment in the MIS group, both groups received similar postoperative radiotherapy doses, with similar rates of local recurrence and hardware failure. An increased ability to perform MIS in emergency settings as well as larger, prospective studies are needed to determine the potential benefits of MIS over standard open separation surgery.

https://thejns.org/doi/abs/10.3171/2021.2.FOCUS201124

KEYWORDS metastatic spinal cord compression; separation surgery; minimally invasive spine surgery; radiotherapy

A DVANCES in systemic therapies for oncological diseases have brought greater attention to a multimodal approach utilizing surgical and radiation treatments for metastatic spinal tumors. Metastatic spinal disease may lead to instability, neurological deficits, and/ or pain due to metastatic epidural spinal cord compression (MESCC). The national incidence of spinal metastases requiring surgery is increasing, along with an overall increase of patient comorbidities and in-hospital complication rates. ${ }^{1}$ Surgery provides immediate spinal stabili-

ABBREVIATIONS KPS = Karnofsky Performance Scale; MESCC = metastatic epidural spinal cord compression; MIS = minimally invasive surgery; RT = radiation therapy; SINS = Spine Instability Neoplastic Score; SRS = stereotactic radiosurgery.

SUBMITTED January 1, 2021. ACCEPTED February 22, 2021.

INCLUDE WHEN CITING DOI: 10.3171/2021.2.FOCUS201124.

${ }^{*}$ M.E. and A.S. share first authorship of this work. 
zation and decompression of the neural elements, while radiation therapy (RT) provides local control. The main treatment goal in these patients remains palliative with regard to symptoms, emphasizing preservation or restoration of spinal stability and neurological function. ${ }^{2}$ As such, treatments are focusing on utilizing less aggressive, minimally invasive techniques.

Standard external-beam RT delivered in fractionated doses successfully provides local control of radiosensitive tumors and has been the mainstay treatment for decades. However, these effects are limited by poor control of radioresistant tumors and risk of radiation-induced injury to the spinal cord with repeat dosing., ${ }^{3,4}$ Alternatively, stereotactic radiosurgery (SRS) allows the delivery of high-dose radiation in a single stage or hypofractionated stages with minimal exposure to surrounding tissues that may be conformed around the spinal cord..$^{5-7}$

With the advent of SRS, the development of separation surgery emerged. Separation surgery-re-creation of a minimum amount of space of 2- to 3-mm margins between the tumor and the spinal cord to allow for safe postoperative SRS-avoids the morbidity of extensive tumor debulking and reconstruction. ${ }^{8-10}$ This strategy has allowed for the exploration of minimally invasive surgery (MIS) approaches with the goals of reducing blood loss, muscle disruption, pain, and recovery time. ${ }^{11}$ Systematic reviews by Pennington et al. and Zuckerman et al. exploring MIS for spinal metastases found that patients treated with MIS tended to achieve these goals with fewer complications. ${ }^{12,13}$ However, the included studies varied significantly with technique, and few focused specifically on comparing MIS versus open separation surgery for MESCC. They also lacked a detailed description of surgical technique. Furthermore, the utility of intraoperative stereotactic navigation in the resection of spinal tumors has been shown, ${ }^{14}$ but its value in MIS separation surgery is still emerging. Here, we provide an overview of our MIS technique for separation surgery with intraoperative stereotactic navigation and compare our experience between open and MIS approaches for providing circumferential separation and allowing safe postoperative SRS.

\section{Methods}

\section{Study Design and Patient Selection}

This is a single-institution, retrospective comparative study of patients undergoing either MIS or standard open surgery for separation and stabilization of spinal metastases. A chart review was performed over a 10-year span between July 2009 and June 2019. Patients with metastatic spinal disease who received circumferential separation via laminectomy and a transpedicular approach to debulk ventral epidural disease as well as instrumented stabilization, through either an open or MIS approach, were included in this study. Patients were excluded from this study if they were younger than 18 years of age and if they underwent more-invasive tumor debulking with anterior column reconstruction, including cage placement, as this was not consistent with the intended goal of separation surgery.

Diagnosis of metastatic disease was confirmed through histopathological analysis performed on intraoperatively obtained specimens. Indications for separation surgery were determined by the NOMS decision framework, which includes the algorithm of neurological, oncological, mechanical, and systemic principles. ${ }^{15}$ Patients with hematological malignancies underwent surgery for neurological deficits due to cord compression with an unknown preoperative diagnosis, including nondiagnostic biopsies. Patients who were diagnosed with solitary plasmacytoma were treated with postoperative radiotherapy with curative intent, and, although their tumors were not radioresistant, the patients were still included in our analysis. ${ }^{16,17}$ Patients were discussed at a multidisciplinary tumor board conference in nonemergency cases.

Collected patient data included age at surgery, sex, presence of medical comorbidities, BMI, preoperative and postoperative Karnofsky Performance Scale (KPS) score, neurological status (assessed by the Frankel scale), Spine Instability Neoplastic Score (SINS), and Tokuhashi score. Operative data included operative time, estimated blood loss, need for red blood cell transfusion, number of levels fused, and postoperative radiation doses. Outcome data included complication rates, 6-month mortality, and neurological status.

\section{Minimally Invasive Tubular Transpedicular Decompression and Percutaneous Instrumentation}

A detailed description of the minimally invasive tubular transpedicular decompression technique has been described previously, including in an operative video. ${ }^{18,19}$ In brief, patients are placed prone on a Jackson table with padding of all pressure points. It is essential that the patient is secured with additional tape and straps to minimize patient movement on the table for navigation accuracy, as well as to allow for more degrees of rotation of the bed to facilitate an increased medial trajectory. A Mayfield head holder is occasionally used for lesions approaching the upper thoracic spine, to allow for use of the rigid arm in fixation of the reference array. In midthoracic cases, an additional incision and spinous process clamp are used. In thoracolumbar and lumbar cases, rigid fixation is obtained using a hip pin. Stereotactic intraoperative navigation is used to plan appropriate paramedian incisions for placement of percutaneous pedicle screws and to determine the entry point for access to the tumor. Pedicle screws are generally placed first, with preference to utilize a system without K-wires to avoid risk of inadvertent advancement, especially in regions of compromised bone integrity.

After pedicle screw placement, a minimally invasive tubular retraction system is inserted under stereotactic guidance. For the thoracic levels, the transverse process is used for docking to provide an appropriately medial trajectory (Fig. 1). After placement of the tubular retractor, we expose the ipsilateral lamina and facet joint. After exposure and identification of the facet joint, the inferior articulating process is removed, exposing the underlying superior articulating process. A bilateral laminotomy via a unilateral approach is performed over the involved level to ensure adequate posterior decompression as well as to visualize the thecal sac (Fig. 2). The superior articulating process is then drilled down to access the foramen, and 

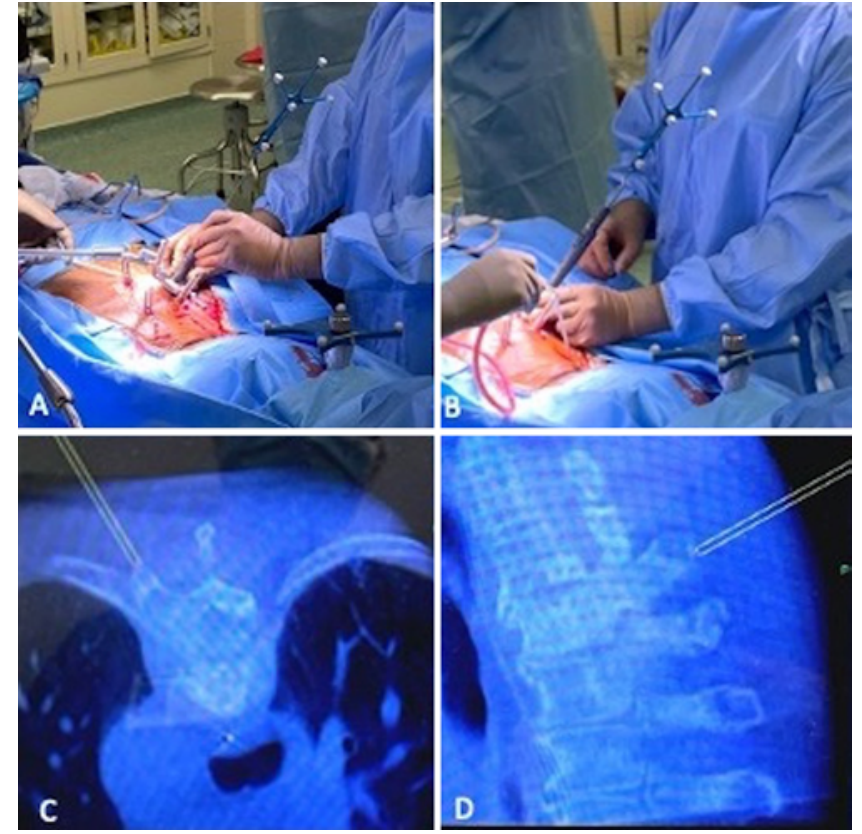

FIG. 1. A: Demonstration of docking with the tubular retractor system using the navigated pointer with the rigid arm. B: Confirmation of final position and removal of tubular dilators. C and D: Real-time axial (C) and sagittal (D) stereotactic images demonstrating docking onto the transverse process of the involved level.

the exiting nerve root is identified. Attention is then turned toward identifying the pedicle at the involved level, and a transpedicular corridor is created to access the epidural space medially and anteriorly. Stereotactic navigation is used to guide the surgeon toward achieving circumferential decompression. Pathologic specimens of the tumor are collected for histological analysis. For decompression anterior to the cord, a partial corpectomy is utilized to create space for a downgoing curette to push the residual tumor away anteriorly into the vertebral body. Care is taken to remain below the posterior longitudinal ligament to avoid any injury to the ventral spinal cord. A Woodson dissector is used to carefully ensure that there is no further ventral compression. A final $\mathrm{O}$-arm image can be obtained to confirm circumferential decompression in the case of osteoblastic bony spinal cord compression (Fig. 3). Rods are placed percutaneously, and satisfactory hardware placement is confirmed by radiography. In cases of osteoblastic lesions requiring only a unilateral approach for sufficient decompression, a short fusion construct was performed.

\section{Statistical Analysis}

Statistical analyses were performed using $\mathrm{R}$ version 4.0.3 (The R Foundation), where the average values for all parameters were compared between groups, and assessed by multivariate ANOVA, with Tukey post hoc analysis for parametric variables and the chi-square test for binary variables. Significance was set at $p<0.05$.

\section{Results}

Characteristics of the 41 patients who underwent either

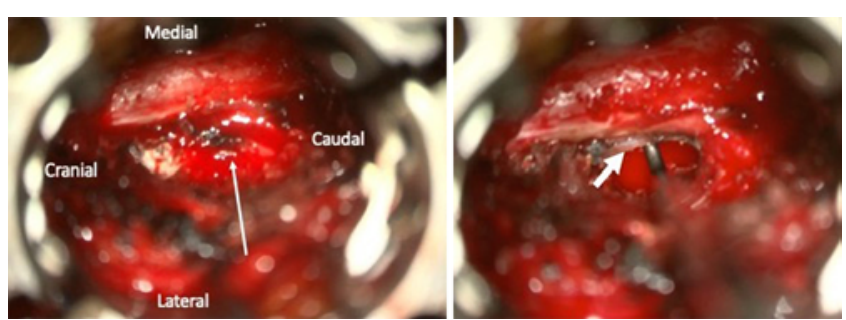

FIG. 2. Left: Intraoperative view after an over-the-top decompression and facetectomies were performed, leaving the involved pedicle (arrow) isolated. Right: A downgoing curette is used below the level of the posterior longitudinal ligament (arrow). A partial corpectomy allows for residual tumor to be pushed away anteriorly into the vertebral body.
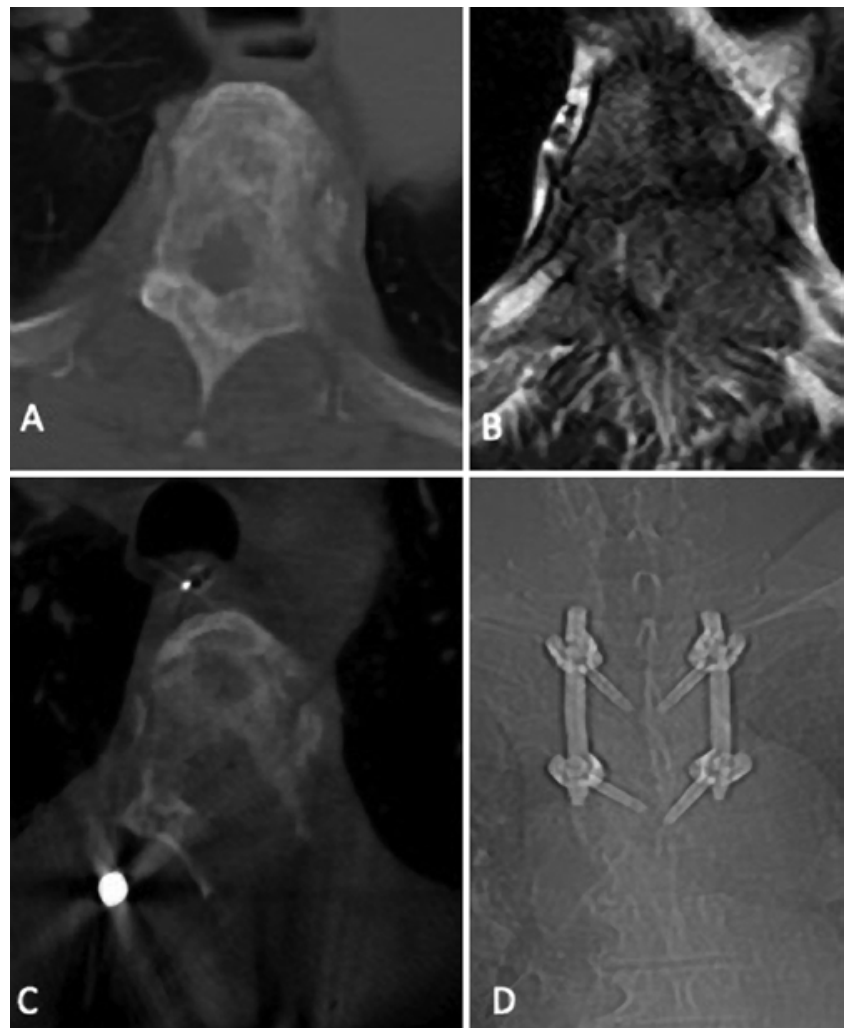

FIG. 3. A: Preoperative CT scan demonstrating blastic prostate metastasis with asymmetrical involvement of the left pedicle. B: Preoperative axial MR image demonstrating high-grade spinal cord compression. C: Intraoperative CT scan demonstrating satisfactory ventral decompression through a unilateral transpedicular approach. D: Postoperative anteroposterior radiograph of percutaneously placed short-segment instrumentation.

MIS or standard open surgery, including both urgent and nonurgent clinical presentations (i.e., more than 48 hours from symptom onset), are presented in Table 1. Pathologies were similar among both groups, including breast, prostate, and lung being the most common, with other diagnoses of plasmacytoma, renal cell carcinoma, hepatocellular carcinoma, cholangiocarcinoma, uterine leiomyosarcoma, colon adenocarcinoma, and adenocarcinoma of unknown origin. There were 17 patients in the MIS group and 24 in the open surgery group. The average age of the 
TABLE 1. Patient characteristics

\begin{tabular}{lccc}
\hline & Open Surgery & MIS & p Value \\
\hline Male sex, \% & 62.5 & 70.6 & 0.84 \\
\hline Mean age, yrs & 56.6 & 65.5 & $0.02^{*}$ \\
\hline Comorbidities, \% & & & \\
\hline Type 2 diabetes & 20.83 & 29.41 & 0.79 \\
\hline Hypertension & 45.83 & 58.82 & 0.62 \\
\hline Coronary artery disease & 4.17 & 17.65 & 0.37 \\
\hline Chronic kidney disease & 12.50 & 11.76 & 1.00 \\
\hline Chronic lung disease & 4.17 & 11.76 & 0.76 \\
\hline Chronic liver disease & 4.17 & 11.76 & 0.76 \\
\hline Mean BMI & 28.4 & 26.4 & 0.19 \\
\hline Mean preop KPS score & 63.0 & 75.9 & $0.02^{*}$ \\
\hline Mean preop Frankel grade† & 4.3 & 4.5 & 0.31 \\
\hline Mean SINS & 11.0 & 9.4 & 0.09 \\
\hline Mean Tokuhashi score & 8.6 & 8.8 & 0.84 \\
\hline * $p<0.05$. & & & \\
\hline Grades A-E are represented as 1-5. & & &
\end{tabular}

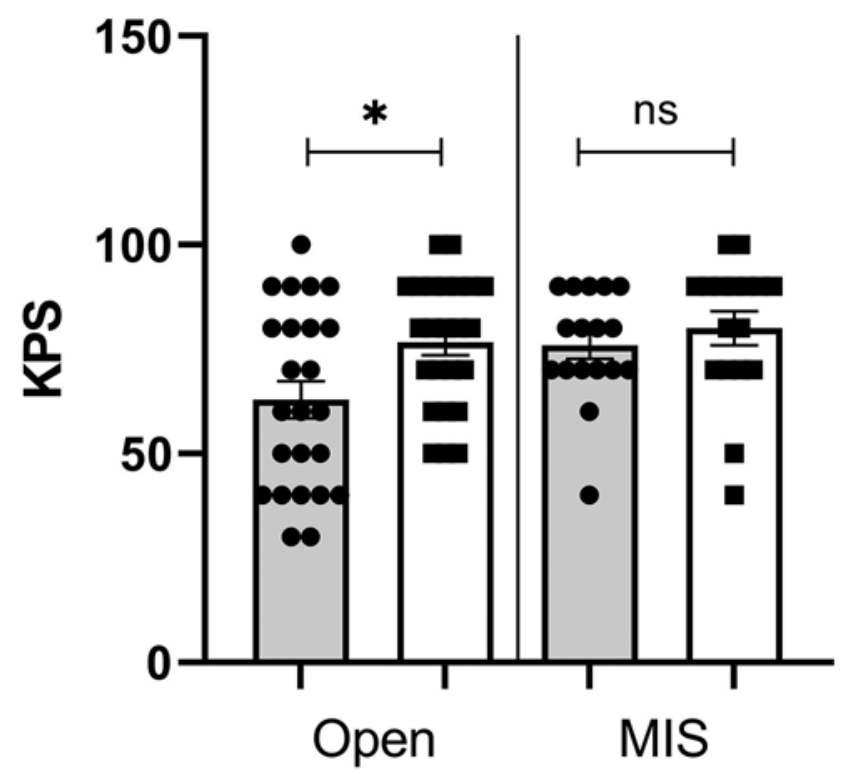

\section{- Pre-Operative - Post-Operative}

FIG. 4. Comparison of preoperative and postoperative KPS scores between open surgery and MIS groups. Bars represent the mean and the error bars the SD. ${ }^{*} p<0.05$; ns = not significant.

MIS group was significantly greater than that of the open surgery group $(65.5$ vs 56.6 years, $p=0.02)$. The preoperative KPS score of the open group was significantly lower than that of the MIS group, with an average of 63.0 versus 75.9 , respectively $(\mathrm{p}=0.02)$, corresponding to an overall less functional population. This was also evidenced by the higher proportion of emergency procedures performed in the open group ( 9 of 24 patients vs 0 of 17 patients, $p=$
TABLE 2. Operative parameters, patient outcomes, and radiotherapy treatment

\begin{tabular}{|c|c|c|c|}
\hline & $\begin{array}{l}\text { Open } \\
\text { Surgery }\end{array}$ & MIS & p Value \\
\hline No. of levels fused & 6.5 & 5.8 & \\
\hline Length of surgery, mins & 405 & 380 & 0.69 \\
\hline Estimated blood loss, $\mathrm{mL}$ & 783 & 430 & $0.02^{*}$ \\
\hline $\begin{array}{l}\text { Packed red blood cells } \\
\text { transfused, } \mathrm{mL}\end{array}$ & 325 & 216 & 0.36 \\
\hline Postop length of stay, days & 12.3 & 13.2 & 0.75 \\
\hline Complications, $\mathrm{n}(\%)$ & $3(13)$ & $1(6)$ & 0.87 \\
\hline 6-mo mortality, n (\%) & $2(8)$ & 0 & 0.63 \\
\hline Postop KPS score & 76.7 & 80.0 & 0.52 \\
\hline Change in KPS score & 13.8 & 4.1 & 0.03 \\
\hline Postop Frankel grade $†$ & 4.7 & 4.6 & 0.74 \\
\hline Change in Frankel grade & 0.43 & 0.18 & 0.23 \\
\hline Postop radiation dose, Gy & $26.5 \pm 6.9$ & $23.5 \pm 5.0$ & 0.084 \\
\hline Days until start of RT & $43.1 \pm 20.3$ & $32.8 \pm 15.6$ & 0.069 \\
\hline \multicolumn{4}{|l|}{$\begin{array}{l}\text { Long-term follow-up } \\
\text { (minimum } 1 \mathrm{yr} \text { ) }\end{array}$} \\
\hline No. of patients & 15 & 16 & \\
\hline Average follow-up, yrs & 2.8 & 4.1 & \\
\hline Local recurrence, n (\%) & $3(20)$ & $2(12.5)$ & \\
\hline
\end{tabular}

0.004). Preoperative comorbidities, BMI, Frankel grade, and Tokuhashi score were similar between the two groups. The SINS was slightly higher in the open group, but not significantly.

Outcomes are summarized in Table 2. The number of levels fused and operative parameters, including length of surgery, were similar, except the average estimated blood loss for the open surgery group was significantly higher than that for the MIS group ( $783 \mathrm{~mL}$ vs $430 \mathrm{~mL}, \mathrm{p}=0.02$ ). However, this did not appear to impact the average amount of packed red blood cells transfused $(325 \mathrm{~mL}$ vs $216 \mathrm{~mL}, \mathrm{p}$ $=0.36$ ). Postoperatively, the open group demonstrated significantly greater improvement in KPS scores, such that the preoperative difference between groups was no longer significant (Fig. 4).

The open surgery group experienced 3 complications, including 2 wound infections and 1 return to the operating room for revision of a pedicle screw, while the MIS group had 1 wound infection. No patients developed radiationrelated wound breakdown. The open surgery group had 2 deaths within 60 days, though these were due to coexisting problems. Finally, postoperatively $23.5 \%$ of patients in the MIS group improved neurologically on the Frankel grade, $64.7 \%$ remained the same, and $11.8 \%$ deteriorated, while $41.7 \%$ of patients in the open group improved, $45.8 \%$ remained the same, and $12.5 \%$ deteriorated (Fig. 5). It is noteworthy, however, that all patients in the MIS group scored Frankel grade D or E, while 6 of the 24 open surgery patients scored Frankel grade C, where the most 

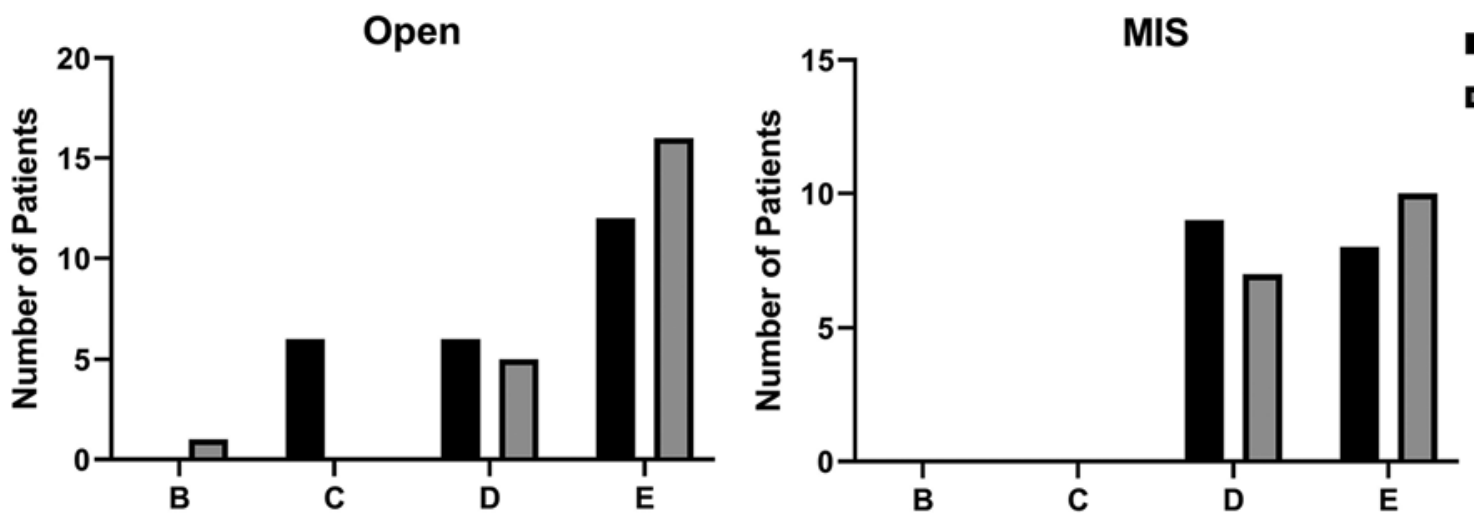

FIG. 5. Comparison of preoperative and postoperative Frankel scale grades between the open surgery (left) and MIS (right) groups.

significant improvement was appreciated. Furthermore, there was no significant difference between groups as pertaining to Frankel grade, in particular with regard to deterioration.

Postoperative radiation doses were available for $18 \mathrm{pa}-$ tients in the open group and 14 patients in the MIS group. In the open surgery group, 12 patients $(66.7 \%)$ received 20-40 Gy in 5-6 fractions, 5 patients $(27.8 \%)$ received 24-30 Gy in 3-4 fractions, and 1 patient (5.5\%) received a single dose of $18 \mathrm{~Gy}$. In the MIS group, 5 patients (35.7\%) received $20-35$ Gy in 5-6 fractions, 8 patients $(57.1 \%)$ received 24-30 Gy in 3-4 fractions, and 1 patient (7.1\%) received a single dose of $24 \mathrm{~Gy}$. The mean dose in the open group was $26.5 \pm 6.9 \mathrm{~Gy}$, and that in MIS was $23.5 \pm 5.0$ Gy $(\mathrm{p}=0.084)$. Time until start of RT was slightly less in the MIS than the open surgery group $(32.8 \pm 15.6$ days vs $43.1 \pm 20.3$ days, $p=0.069$ ). This included 4 patients in the MIS group who underwent RT during the same admission as surgery.

Long-term results (a minimum of 1-year follow-up) were available for the majority of patients (Table 2). In the open surgery group, 3 of the 15 (20\%) patients with long-term follow-up were found to have recurrence at the site of surgery with MESCC compared with 2 of the 16 (12.5\%) patients treated with MIS technique. Two of these recurrences arose in patients with highly malignant uterine leiomyosarcomas, and the others occurred in hepatocellular, renal cell, and colon adenocarcinoma. No patient in either group developed hardware failure requiring revision surgery.

\section{Discussion}

The term "separation surgery" is credited to Lilyana Angelov and Edward Benzel at the Cleveland Clinic to designate a procedure in which tumor resection is limited to decompression of the spinal cord to create enough space to provide a safe target for spine SRS. ${ }^{20}$ Moulding et al. introduced the concept to the literature in 2010 by demonstrating the effectiveness of high-dose adjuvant SRS after surgical decompression and stabilization to achieve local disease control and preserve neurological function. ${ }^{21}$ As such, the integration of limited cytoreductive surgery with postoperative radiosurgery in the treatment of spinal metastatic tumors has led to a shift in surgical goals..22 Moreinvasive surgeries that carry increased risk of complications and surgical morbidity, such as staged thoracotomy or retroperitoneal approaches, corpectomies with anterior column reconstruction, or spondylectomies with wide excision, are now less frequently utilized..$^{10,23,24}$ Because of the advancements in technique, including less local tissue disruption and a potentially faster rate of recovery while maintaining safe targets for high-dose radiotherapy, MIS has become the new state-of-the-art treatment for MESCC. ${ }^{8,25,26}$ In this study, we have demonstrated that, although there was a trend toward faster time to starting radiation treatment in the MIS group, there were similar postoperative radiotherapy doses among both groups, with no increased rate of local recurrence and no occurrences of hardware failure.

Furthermore, the prediction of patient outcomes regarding survival and quality of life after surgery remains challenging despite numerous prognostic scores that attempt to stratify patients into risk groups. ${ }^{27-30}$ MIS techniques may be able to expand our patient population to include those who are considered candidates for surgery despite stratification into a higher risk group. In recent studies, patients with metastatic spinal disease treated with MIS approaches tended to have decreased blood loss, shorter inpatient stays, and fewer complications compared with patients undergoing open procedures while maintaining similar outcomes in regard to pain, neurological improvement, and function. ${ }^{12}$ The results presented herein demonstrate overall noninferiority of MIS as compared with open separation surgery for metastatic spinal disease despite a tendency toward use of the latter in an older population.

Similar to other studies, our results were biased against using MIS techniques in patients with emergency presentation, i.e., within 24 hours of developing profound weakness. Lau and Chou also reported that the time of surgery dictated the choice of mini-open versus an open approach; scheduled cases during elective hours had the necessary staff and navigation support needed for MIS, whereas patients presenting at night or on the weekend were relegated to an open approach. ${ }^{31}$ Conversely, Miscusi et al. demon- 
TABLE 3. Candidates for minimally invasive separation surgery

\begin{tabular}{|c|c|c|}
\hline Potential Candidates & Contraindications & Relative or Institutional Considerations \\
\hline $\begin{array}{l}\text { Access to a multidisciplinary spinal oncology team } \\
\text { offering postop adjuvant SRS }\end{array}$ & Life expectancy $<3$ mos & Older age or multiple medical comorbidities \\
\hline Life expectancy $>3$ mos & More than 2-level involvement & Access to intraoperative stereotactic navigation \\
\hline 1- or 2-level involvement & \multirow{2}{*}{$\begin{array}{l}\text { Anterior \& middle column involvement or } \\
\text { need for anterior column reconstruction* }\end{array}$} & \multirow{2}{*}{$\begin{array}{l}\text { Emergency presentation requiring off-hours } \\
\text { (night or weekend) surgical intervention }\end{array}$} \\
\hline Absence of gross instability denoted by a SINS $<13^{*}$ & & \\
\hline
\end{tabular}

* In the presence of a pathologic fracture or mechanical instability where anterior column reconstruction is required, an open or mini-open approach for transpedicular corpectomy and anterior cage placement should be employed.

strated an effective use of the MIS technique for patients with acute presentation due to high-grade MESCC causing myelopathy, with a significant improvement in terms of blood loss, operation time, days in bed, and decreased length of stay compared with a retrospective control group of patients who underwent open surgery. ${ }^{32}$ In a previous prospective propensity score-matched analysis, the MIS group again was found to have the advantage of less blood loss, lower rate of blood transfusion, lower average units of blood transfused, and shorter hospital stays. ${ }^{33}$

Our results demonstrate a significantly lower average estimated blood loss for the MIS group; however, this did not appear to significantly impact the average amount of packed red blood cells transfused or the length of stay. Of note, all abovementioned studies included patients who required anterior column reconstruction, performed via either open surgery or MIS, which was often through a mini-open or hybrid approach. ${ }^{31-33}$ As such, our results, which focused on separation surgery with only limited resection and stabilization among both groups, demonstrated a total lower rate of blood loss, need for blood transfusion, and postoperative length of stay for both open and MIS groups compared with the results of prior studies. Additionally, length of stay in our MIS cohort was confounded by several patients receiving inpatient RT during the same admission as surgery.

Another factor that may have influenced blood loss is the nature of performing a limited resection. Typically, bleeding is better controlled once the majority of the tumor is removed; however, this is by definition not the goal in separation surgery. Here, the objective is to create sufficient space around the neural elements to allow for safe postoperative RT and not a gross-total resection. This means blood loss must be well controlled throughout the surgery, especially for vascular tumors like hepatocellular and renal cell carcinoma. Hemostasis is made even more difficult during MIS access with a limited surgical field. Considerations for preoperative embolization should always be discussed with the interventional radiologist, ${ }^{34}$ and, at our institution, all hypervascular tumors are embolized. In addition, we use a series of hemostatic maneuvers to control bleeding. A 4- or 6-mm coarse diamond burr is used during creation of the transpedicular corridor and partial corpectomy as it cauterizes while drilling, which aids in hemostasis. ${ }^{19}$ A combination of Avitene, Gelfoam, and other thrombin-mixed hemostatic agents can also be very useful. This may require several rounds of application before adequate control is achieved. In addition, we gener- ally overlay the tumor edges with Surgicel at the end of the procedure. As the degree of tumor resection between the two techniques was similar, the MIS technique is able to achieve significantly less blood loss through minimizing bleeding during exposure.

Stereotactic navigation is becoming more widespread in its utilization and can be a valuable intraoperative adjunct. ${ }^{35}$ Its effectiveness during MIS to allow surgeons greater understanding of bony and soft-tissue anatomy through limited incisions and exposure, as well as minimalizing radiation exposure, has been demonstrated. ${ }^{23,36}$ Its unique contribution during MIS separation surgery lies in the abnormal anatomy inherent to tumoral invasion. ${ }^{14,18,37}$ Thus, the use of guidance to identify the abnormal anatomy and determine the extent of resection is indispensable. Final imaging with intraoperative CT scanning can verify complete ventral decompression. Additionally, navigation offers higher accuracy in the placement of pedicle screws, specifically for patients with metastatic disease who often have poor bone quality and are potentially at higher risk of pedicle breach or fractures. ${ }^{38}$ The limited perspective of a tubular retractor system without stereotactic navigation, and fluoroscopically placed K-wires for percutaneous pedicle screws in the setting of metastatic spinal infiltration with abnormal and poor bony integrity, warrants reconsideration.

The data presented herein demonstrate overall equivalent perioperative complication and morbidity of MIS as compared with open separation surgery for MESCC. There are, however, certain limitations to this study. The main drawbacks are related to the retrospective nature of this study as well as the limited cohort. There was also selection bias in performing emergency surgery with a conventional open approach, which resulted in a lower preoperative functional performance score in the open group as well as potentially slightly higher rate of complications. Additionally, the open group tended to have higher SINS, more levels fused, and the surgery was more likely to cross a junction, either cervicothoracic or thoracolumbar. Table 3 lists considerations for best candidates for MIS separation surgery according to various reports and qualitative reviews. ${ }^{12,13,27,39,40}$ Other variables, such as opioid consumption, days in bed, pain scores, and quality-of-life patientreported outcome measures, were not available in our retrospective review. These factors may have been influenced by the choice of open or MIS approach but unfortunately were unable to be assessed. However, our results uniquely demonstrate the potential for MIS separation surgery to 
decrease morbidity and allow for the faster delivery of RT with similar rates of local control and stabilization compared with open separation surgery.

\section{Conclusions}

MIS separation surgery for metastatic MESCC is a safe and effective approach for decompression and stabilization compared with standard open separation surgery, and it significantly reduced blood loss during surgery. Although there was a trend toward a faster time to starting radiation treatment in the MIS group, both groups received similar postoperative radiotherapy doses, with similar rates of local recurrence and hardware failure. An increased ability to perform MIS in emergency settings as well as larger, prospective studies are needed to determine the potential benefits of MIS over standard open separation surgery.

\section{References}

1. Yoshihara H, Yoneoka D. Trends in the surgical treatment for spinal metastasis and the in-hospital patient outcomes in the United States from 2000 to 2009. Spine J. 2014;14(9): 1844-1849.

2. Barzilai O, Fisher CG, Bilsky MH. State of the art treatment of spinal metastatic disease. Neurosurgery. 2018;82(6): 757-769.

3. Gerszten PC, Ozhasoglu C, Burton SA, et al. CyberKnife frameless stereotactic radiosurgery for spinal lesions: clinical experience in 125 cases. Neurosurgery. 2004;55(1):89-99.

4. Wara WM, Phillips TL, Sheline GE, Schwade JG. Radiation tolerance of the spinal cord. Cancer. 1975;35(6):1558-1562.

5. Benzil DL, Saboori M, Mogilner AY, et al. Safety and efficacy of stereotactic radiosurgery for tumors of the spine. $J$ Neurosurg. 2004;101(suppl 3):413-418.

6. Gerszten PC, Welch WC. Cyberknife radiosurgery for metastatic spine tumors. Neurosurg Clin N Am. 2004;15(4): 491-501.

7. Medin PM, Solberg TD, De Salles AAF, et al. Investigations of a minimally invasive method for treatment of spinal malignancies with LINAC stereotactic radiation therapy: accuracy and animal studies. Int J Radiat Oncol Biol Phys. 2002; 52(4):1111-1122.

8. Moussazadeh N, Laufer I, Yamada Y, Bilsky MH. Separation surgery for spinal metastases: effect of spinal radiosurgery on surgical treatment goals. Cancer Contr. 2014;21(2):168-174.

9. Bate BG, Khan NR, Kimball BY, et al. Stereotactic radiosurgery for spinal metastases with or without separation surgery. J Neurosurg Spine. 2015;22(4):409-415.

10. Barzilai O, Bilsky MH, Laufer I. The role of minimal access surgery in the treatment of spinal metastatic tumors. Global Spine J. 2020;10(2)(suppl):79S-87S.

11. Fessler RG, O'Toole JE, Eichholz KM, Perez-Cruet MJ. The development of minimally invasive spine surgery. Neurosurg Clin N Am. 2006;17(4):401-409.

12. Pennington $Z$, Ahmed AK, Molina CA, et al. Minimally invasive versus conventional spine surgery for vertebral metastases: a systematic review of the evidence. Ann Transl Med. 2018;6(6):103.

13. Zuckerman SL, Laufer I, Sahgal A, et al. When less is more: the indications for MIS techniques and separation surgery in metastatic spine disease. Spine (Phila Pa 1976). 2016;41(12) (suppl 20):S246-S253.

14. Nasser R, Drazin D, Nakhla J, et al. Resection of spinal column tumors utilizing image-guided navigation: a multicenter analysis. Neurosurg Focus. 2016;41(2):E15.

15. Laufer I, Rubin DG, Lis E, et al. The NOMS framework: ap- proach to the treatment of spinal metastatic tumors. Oncologist. 2013;18(6):744-751.

16. Miller JA, Balagamwala EH, Chao ST, et al. Spine stereotactic radiosurgery for the treatment of multiple myeloma. $J$ Neurosurg Spine. 2017;26(3):282-290.

17. Tsang RW, Gospodarowicz MK, Pintilie M, et al. Solitary plasmacytoma treated with radiotherapy: impact of tumor size on outcome. Int J Radiat Oncol Biol Phys. 2001;50(1): 113-120.

18. Nasser R, Nakhla J, Echt M, et al. Minimally invasive separation surgery with intraoperative stereotactic guidance: a feasibility study. World Neurosurg. 2018;109:68-76.

19. De la Garza Ramos R, Echt M, Gelfand Y, et al. Minimally invasive tubular separation surgery for metastatic spinal cord compression: 2-dimensional operative video. Oper Neurosurg (Hagerstown). Published online December 29, 2020. doi: 10.1093/ons/opaa421

20. Spratt DE, Beeler WH, de Moraes FY, et al. An integrated multidisciplinary algorithm for the management of spinal metastases: an International Spine Oncology Consortium report. Lancet Oncol. 2017;18(12):e720-e730.

21. Moulding HD, Elder JB, Lis E, et al. Local disease control after decompressive surgery and adjuvant high-dose singlefraction radiosurgery for spine metastases. J Neurosurg Spine. 2010;13(1):87-93.

22. Di Perna G, Cofano F, Mantovani C, et al. Separation surgery for metastatic epidural spinal cord compression: a qualitative review. J Bone Oncol. 2020;25:100320.

23. Hussain I, Fu KM, Uribe JS, et al. State of the art advances in minimally invasive surgery for adult spinal deformity. Spine Deform. 2020;8(6):1143-1158.

24. Laufer I, Bilsky MH. Advances in the treatment of metastatic spine tumors: the future is not what it used to be. J Neurosurg Spine. 2019;30(3):299-307.

25. Barzilai O, Laufer I, Robin A, et al. Hybrid therapy for metastatic epidural spinal cord compression: technique for separation surgery and spine radiosurgery. Oper Neurosurg (Hagerstown). 2019;16(3):310-318.

26. Joaquim AF, Powers A, Laufer I, Bilsky MH. An update in the management of spinal metastases. Arq Neuropsiquiatr. 2015;73(9):795-802.

27. Arrigo RT, Kalanithi P, Cheng I, et al. Predictors of survival after surgical treatment of spinal metastasis. Neurosurgery. 2011;68(3):674-681.

28. Schoenfeld AJ, Leonard DA, Saadat E, et al. Predictors of 30- and 90-day survival following surgical intervention for spinal metastases: a prognostic study conducted at four academic centers. Spine (Phila Pa 1976). 2016;41(8):E503-E509.

29. Choi D, Fox Z, Albert T, et al. Prediction of quality of life and survival after surgery for symptomatic spinal metastases: a multicenter cohort study to determine suitability for surgical treatment. Neurosurgery. 2015;77(5):698-708.

30. Tokuhashi Y, Ajiro Y, Umezawa N. Outcome of treatment for spinal metastases using scoring system for preoperative evaluation of prognosis. Spine (Phila Pa 1976). 2009;34(1): 69-73.

31. Lau D, Chou D. Posterior thoracic corpectomy with cage reconstruction for metastatic spinal tumors: comparing the mini-open approach to the open approach. J Neurosurg Spine. 2015;23(2):217-227.

32. Miscusi M, Polli FM, Forcato S, et al. Comparison of minimally invasive surgery with standard open surgery for vertebral thoracic metastases causing acute myelopathy in patients with short- or mid-term life expectancy: surgical technique and early clinical results. J Neurosurg Spine. 2015;22(5): $518-525$.

33. Hansen-Algenstaedt N, Kwan MK, Algenstaedt P, et al. Comparison between minimally invasive surgery and conventional open surgery for patients with spinal metastasis: a 
prospective propensity score-matched study. Spine (Phila Pa 1976). 2017;42(10):789-797.

34. Hong CG, Cho JH, Suh DC, et al. Preoperative embolization in patients with metastatic spinal cord compression: mandatory or optional? World J Surg Oncol. 2017;15(1):45.

35. Kochanski RB, Lombardi JM, Laratta JL, et al. Image-guided navigation and robotics in spine surgery. Neurosurgery. 2019;84(6):1179-1189.

36. Virk S, Qureshi S. Navigation in minimally invasive spine surgery. J Spine Surg. 2019;5(suppl 1):S25-S30.

37. Kelly PD, Zuckerman SL, Yamada Y, et al. Image guidance in spine tumor surgery. Neurosurg Rev. 2020;43(3):10071017.

38. De la Garza Ramos R, Echt M, Benton JA, et al. Accuracy of freehand versus navigated thoracolumbar pedicle screw placement in patients with metastatic tumors of the spine. $J$ Korean Neurosurg Soc. 2020;63(6):777-783.

39. Alamanda VK, Robinson MM, Kneisl JS, Patt JC. Functional and survival outcomes in patients undergoing surgical treatment for metastatic disease of the spine. J Spine Surg. 2018; 4(1):28-36.

40. Pennington Z, Sciubba DM. Commentary: Minimallyinvasive tubular separation surgery for metastatic spinal cord compression. Oper Neurosurg (Hagerstown). Published online January 11, 2021. doi:10.1093/ons/opaa438

\section{Disclosures}

The authors report no conflict of interest concerning the materials or methods used in this study or the findings specified in this paper.

\section{Author Contributions}

Conception and design: Yassari, Echt, Stock, Nasser.

Acquisition of data: Echt, Hamad, Holland, Nasser. Analysis and interpretation of data: Echt, Stock, Der. Drafting the article: Yassari, Echt, Stock, De la Garza Ramos, Hamad, Holland, Cezayirli. Critically revising the article: Yassari, Echt, Stock, De la Garza Ramos, Cezayirli, Nasser, Yanamadala. Reviewed submitted version of manuscript: Yassari, Echt, Stock, De la Garza Ramos, Yanamadala. Statistical analysis: Echt, Stock, De la Garza Ramos, Der. Administrative/technical/material support: Yassari. Study supervision: Yassari.

\section{Correspondence}

Reza Yassari: Montefiore Medical Center/Albert Einstein College of Medicine, Bronx, NY. ryassari@montefiore.org. 